\title{
On Block Design and the Special Class of (123)-avoiding Aunu Permutation Pattern: A Slanted Flag Model
}

\author{
*Ibrahim M., "Ibrahim A. A., and "Magami M. S \\ "Mathematics Department Usmanu Danfodiyo University Sokoto, P.M.B.2346 Sokoto,Nigeria.
}

\begin{abstract}
This paper concerns an important application of the (123)-avoiding class of Aunu Permutation studied by earlier by Usman, Magami and Garba under various topics such as enumeration, thin cyclic desighn and association schemes. In this report, some method of construction are used using accents and descents as enumerated by positive and negative units in a manner that resembles, in some sense, the Motzkin numbers. This method produced some graph models which when superimposed produced the required design of a slanted flag. This paper therefore uncovers yet another rich property of this class of permutation pattern: applications in design theory which interested researchers can further explore.
\end{abstract}

Key Words: Design theory, Permutation, avoiding pattern, Flag Algorithm, Special class.

\section{Introduction}

The special classes of (123)-avoiding and (132)-avoiding permutation patterns were first reported in [1] in connection with some group theoretic applications. Subsequent communications which were mainly centered on the (123)-avoiding type could be found in [2], which explained the procedure for generating the sucession scheme for the pattern, together with some graph theoretic consequences. In later repots, applications were identified in coding theory [3]; number theory [4] and in automata theory as well as circuit design and circuit analysis, [5]. It also has identified and discussed some other theoretical properties of the Aunu Patterns and Aunu Groups especially in relation to integer modulo arithmetics which was further extended to produce an integer sequences. [6] The concept of Catalan numbers was also used in comparison, to develop the scheme for prime cardinalities $P^{3} 5$ of arbitrary WÍ $\square$ which generate the cycle of Aunu patterns as detailed in [7]. Further, some construction process was undertaken of some association schemes using Aunu permutation patterns which avoid (123) as well as examination grading system, $A$ to $F$. This method was further extended in [8] and [9] in relation to association schemes of the first kind an investigation was carried out on whether or not the parameters of the first kind and the equivalence relation. We adopt a strict definition of systematic, insisting that the computations can be performed without any human interaction whatsoever. For the definition of enumeration, we used algorithm to compute the number of length $n$ permutations in the class. [10]. This paper therefore, identified a connection between the method of succession as used in generating cyclic structures in [2] and associated scheme. [11]

On the other hand, the special (132)-avoiding class was used in the construction of Latin squares as in [3], thus provoking some design theoretic applications. This paper, exposes for the first time, a useful application in the design theory involving this class of (123)-avoiding permutation pattern.

Further, this paper adopts the use of a variety of n-skip calculable engine to derive transition states which are later modeled into graph using a path tracing procedure comparable with motzkin, [6]. The concept of calculable engines can be traced back to the time of Alan Turing [7] who built an idealized model for mathematical calculation. Later researches had centered on the variability of both the state of the system, expressed as 'head' and the corresponding function $d$.

\section{Some Basic Notions And Notation}

In order to make this paper more self contained, some basic concepts as are related to the paper are supplied together with notation adopted in the construction process of the theoretic frameworks which are central to the main themes of this paper.

\section{Design theory}

There are different types of designs in design theory. These include block design cyclic design, symmetric designs, and the rest. All designs are based on association scheme from which they derive. An association scheme concerns relation between pairs of elements of a non empty set

$$
\mathrm{W}=\{1,2, \ldots\}
$$

where in this case Wis necessarily finite . 
But association schemes are also defined relative to the work they do in a given theoretic scheme. One such association scheme can be defined relative to a 5-element sample

$$
A_{5}=\{1,2,3,4,5\}
$$

where some axioms were defined [11] governing both the nature and the degree of association

$$
A_{i}=\left\{i \mathrm{Q} j: i, j \hat{\mathrm{I}} A_{5}, j=(i+1), \ldots(i+4)\right\}
$$

which depends on some precedence parameter $\mathrm{Q}_{i}$ as in [2] .

On the other hand, a block design on $\mathrm{W}$ for treatment-set $\mathrm{Q}$ is a function $i: \mathrm{W} \otimes \mathrm{Q}$ where $\mathrm{W}$ is the set of plots while $Q$ is the set of treatments and $i$ is the design function. Since there is inherent need for association between subsets of $\mathrm{W}$, the concept of group divisible association scheme comes to play some role. Thus, we also define $\mathrm{Q}$ as the group divisible association scheme defined on W. Consequently, we write

$$
\tilde{\mathrm{A}}:(w, u)=\frac{\dot{1} 1}{1} \quad \text { if } u(w)=0
$$

Then A defines the design matrix in $\hat{\mathrm{A}}^{\mathrm{W} \mathrm{Q}}$.

If we now let $\mathrm{C}$ as the set of blocks in $\mathrm{W}$, the incidence matrix $\mathrm{M}$ in $\hat{\mathrm{A}}^{\mathrm{W} \mathrm{Q}}$ is given by

$$
\mathrm{M}(g, u)=|\{w \hat{\mathrm{I}} g \mid i(w)=u\}|
$$

\section{Accent}

This represents a situation where

$$
\left(a_{i}-a_{j}\right)=1 ; a_{i}, a_{j} \hat{\mathrm{I}} C_{n}
$$

Where $C_{n}$ represents the nth succession in accordance with ()

\section{Descent}

By descent is meant the opposite of (1) so that

$$
\left(a_{i}-a_{j}\right)=-1 ; a_{i}, a_{j} \hat{\mathrm{I}} C_{n}
$$

\section{(123)-avoiding Path}

A path can be defined as any sequence of edges connected in such a manner that no edge or vertex is repeated. In the (123)-avoiding path, it is required that vertices are joined diagonally by edges in square grid so that accents and descents are as defined by the preceding relations,(4) and (5).

When these paths are superimposed in a two-dimensional plane the required model is obtained.

\section{Method Of Construction}

The following represents an algorithm for the construction of the model.

Step 1: For $n^{3} 1, i £ 5 d o$

Step 2: $n \neg n+i$

Step 3: Until $i=4$

Step 4: If $n=5, n+i \neg i$

Step 5: Halt when $n+i=i$

Step 6: Repeat Step 2 for $j=i+1, \ldots, i+(n-2)$

End

\section{The emerging Model}

The aforementioned algorithm gives rise to a total of (5-1) transition diagrams one each from the transition function 


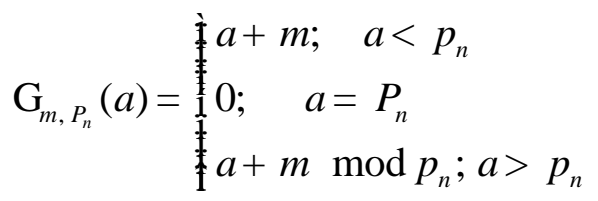

defined in Ibrahim [5] where, in this case $P_{n}=5$, while $i=m ; a=n$.

Thus, for $i=1$, the transition diagram based on some calculable engine can be represented as:

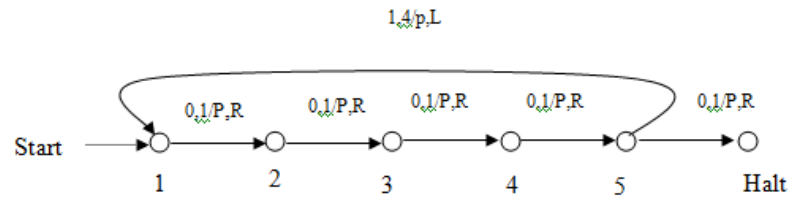

The remaining three transition diagrams can be constructed similarly using $i=2 ; i=3 ; i=4$ in (3). Now denoting as vertices, the end points $n, n+1, \ldots n+(5-1)$ and as edges, the lines joining them, four paths are traced in this 5-element sample as follows:
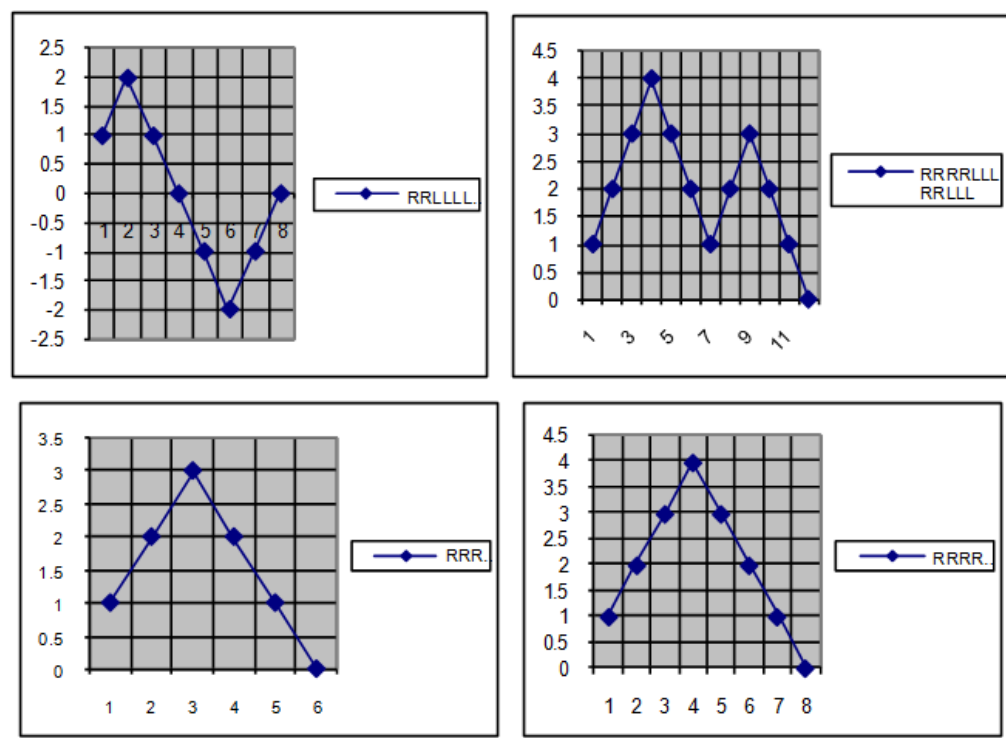

Fig. 1: The four paths traced by this model in accordance with a transition scheme governed by a class of (123)avoiding pattern

Note: in Fig. 1, R represents movement of the tape rightwards in the calculable engine which is also represents an accent/upward motion but diagonally across grid cells in the path tracing graph models. Similarly the later L denotes a leftwards motion, herein called the descent which represents a downwards shift, one square grid diagonally in the path tracing models.

Now, on transposing the graph paths of Fig 1, we have:

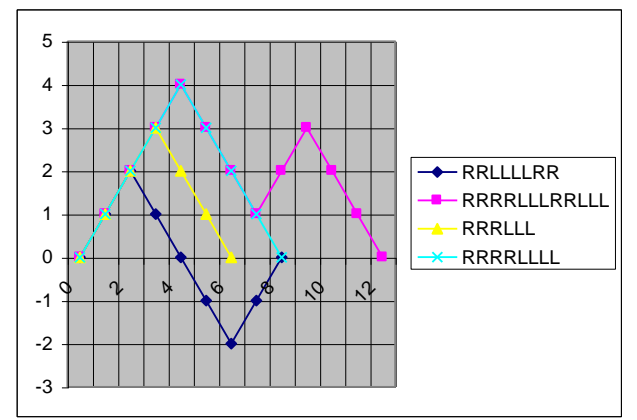

Fig. 2: The Slanted flag model 
Note that due to superimposition of the four different colours, the pink colour is less pronounced on the flag. However it is well pronounced on the shadow of the slanting flag

\section{Discussion Of Important Results}

In contrast with the Motzkin paths, this scheme allows for values of descents below zero level along yaxis. This provides an unrestricted application in two-dimensional plane for the design models arising from this scheme. The resulting model also enumerates the number of paths according to this scheme as realizable from this class of (123)-avoiding pattern of permutation.

It therefore follows that since this scheme is only defined for sets of prime size, Ibrahim [2], this number is clearly given by

$$
N(\text { paths })_{(123)-\text { avioding }}=\left(P_{n}-1\right)
$$

Where $P_{n}$ represents an nth prime which, in this case if 5 .

\section{Conclusion}

In this paper, a five element sample is used to construct the slanting flag model using on a database package: the Microsoft excel. This package was deliverately selected because of the ease of assigning colours such that bioth the vertices and arcs of the corresponding graph models were assigned different colours in order to provide quick visual identification of the models. This paper therefore has oped up another interdisciplinary approach to the study of this permutation pattern by providing an application area in design theory.

\section{Recommendation And Open Problem}

It should be noted however, that the transition scheme based on which the model was built is not restricted only to sets of size five. Further researches should therefore try to discover applications when $P_{n}>5$ . On the other hand, number theoretic applications should be investigated using decomposition schemes and also by enumerating the number of subsequences arising from these patterns.

\section{References}

[1]. Ibrahim A.A and Audu M.S. (2005) Some Group theoretic Properties of Certain Class of (123) and (132)-Avoiding Patterns Numbers: an enumeration scheme African journal Natural Sciences Vol. 8: 79-84

[2]. Ibrahim A.A. (2005) On the Combinatorics ofn Succession Using a 5-Element Sample. Abacus, Journal of Mathematics Association of NIgeria., .32(2B):410-415

[3]. Ibrahim A.A. (2008) Some Transformation Schemes Involving the Special (132) - avoiding Permutation Patterns and a Binary Coding: An Algorithmic Approach Asian Journal of Algebra 1 (1):10-14, Asian Network for Scientific Information (ANSI)

[4]. Sloane N.J.S (2006) Integer Sequence A119626 Online Encyclopeadia of Integer sequence, http://www.research.att.com/ njas/sequence/?q=119626\&sort=0\&language=english\&go=search

[5]. Ibrahim A.A and Isah G.A (2010) On the Use of Automat Models in the Study of a Class of (123)-avoiding Permutation Patterns: Applications in Circuit Design and in Number Theory (In process)

[6]. Usman A. and Ibrahim A. A. (2011b), On Combiantional Interpretations of Aunu Patterns. Katsina Journal of Pure and Applied Sciences. 2(1):161-165

[7]. Usman A. and Ibrahim A. A. (2011a), A new Generating function for Aunu Patterns: Application in Integer Group Modulo n. Nigeria Journal of Basic and Applied Sciences 19(1): 1-4

[8]. Magami M.S and Ibrahim A.A (2011), Construction of some Varieties of association scheme; Appicationa in Typical Examination grading system. Mathematical Association of Nigeria proceeding of Annual National Conference: 33-37

[9]. Magami M.S and Ibrahim A.A (2011),Construction an equivalence relation using Association scheme of the first kind. Proceedings of international conference on science and sustainable development, 2(1): 68-71

[10]. Usman A. and Ibrahim A. A. (2011), Enumeration scheme of (123)-avoiding pattern to Aunu Group, Using Thin Cyclic Design. Mathematical Association of Nigeria proceeding of Annual National Conference: 33-37

[11]. 11 Magami M.S and Ibrahim A.A (2011) Construction of association scheme using some (123)-avoiding class of Aunu permutation patterns. Nigeria Journal of Basic and Applied Sciences 19(1): 5-8 